\title{
Circulating KL-6, a biomarker of lung injury, in obstructive sleep apnoea
}

\author{
D.J. Lederer*, S. Jelic*, R.C. Basner*, A. Ishizaka* and J. Bhattacharya*
}

ABSTRACT: In obstructive sleep apnoea (OSA), oxidative stress contributes to endothelial dysfunction in the peripheral circulation. In the lung, oxidative stress can lead to alveolar injury. The present authors hypothesised that patients with OSA would have biomarker evidence of increased alveolar wall permeability.

Sleep characteristics, brachial artery flow-mediated dilation and plasma KL-6 levels were observed in 11 otherwise healthy patients with OSA and 10 controls.

Median (interquartile range) plasma KL-6 levels were higher in patients with OSA compared with controls: $317(232-506) \mathrm{U} \cdot \mathrm{mL}^{-1}$ versus 226 (179-257) $\mathrm{U} \cdot \mathrm{mL}^{-1}$, respectively. Higher plasma KL-6 levels were associated with greater time spent asleep with an oxyhaemoglobin saturation $<\mathbf{9 0 \%}$, lower nadir saturation, more frequent desaturation of $>\mathbf{4} \%$ during sleep and lower brachial artery flow-mediated dilation. Adjustment for nadir saturation or flow-mediated dilation attenuated the association between plasma KL-6 levels and OSA.

Circulating KL-6 levels are elevated in some patients with obstructive sleep apnoea, possibly reflecting increased alveolar wall permeability.

KEYWORDS: Alveolar wall permeability, biological markers, hypoxia, obstructive sleep apnoea

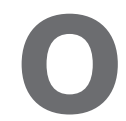

bstructive sleep apnoea (OSA), a chronic disorder characterised by sleep-related upper airway obstruction [1], is associated with increased oxidative stress, which contributes to endothelial dysfunction in the peripheral circulation [2]. However, the injurious effects of oxidative stress in the lungs of patients with OSA are largely unknown.

Oxidative stress in the alveolus can lead to epithelial and endothelial cell injury [3], which increases permeability of the alveolar wall and, when severe, causes pulmonary oedema [4]. While pulmonary oedema occurs routinely in a dog model of OSA [5], it has only rarely been reported in humans with OSA [6]. Therefore, if OSA injures the alveolus, such injury would be expected to be mild, leading to only modest increases in alveolar wall permeability. The present authors hypothesised that it would be possible to detect subclinical lung injury in OSA by measuring circulating levels of KL-6, a mucinlike integral membrane glycoprotein localised to type-II alveolar epithelial cells and which is consistently elevated in the blood of patients with lung injury [7-9].

\section{METHODS}

\section{Study design and subjects}

The present authors retrospectively studied 11 OSA patients and 10 controls enrolled in a prospective study at Columbia University (New York, NY, USA) [2]. Briefly, patients with newly diagnosed OSA, defined as an apnoea/hypopnoea index (AHI) of $\geqslant 5$ obstructive events per hour of sleep, and free of conditions known to affect the vascular endothelium, were eligible for the prospective study. Exclusion criteria included cardiovascular disease or risk factors, pulmonary disease, recent smoking and use of medications [2].

Control subjects were nonsmoking healthy adults recruited from the community through advertising and matched to OSA patients by sex, age ( \pm 4 yrs) and body mass index (BMI; $\pm 15 \%)$. All control subjects had a normal physical examination and laboratory tests and underwent nocturnal polysomnography.

In the current study, all the study subjects who provided plasma during participation in the prospective study were included. The Columbia University Medical Center Institutional Review Board approved the study.
AFFILIATIONS

*Dept of Medicine, Columbia

University, New York, NY, USA, and "Dept of Medicine, Keio University School of Medicine, Tokyo, Japan.

CORRESPONDENCE

D.J. Lederer

Division of Pulmonary, Allergy and Critical Care Medicine

Columbia University College of

Physicians and Surgeons

622 W 168th St

$\mathrm{PH}-8$

Room 101

New York

NY 10032

USA

Fax: 12123422101

E-mail: DL427@columbia.edu

Received:

October 052008

Accepted after revision:

December 152008

SUPPORT STATEMENT

D.J. Lederer received funding from the National Institutes of Health (Bethesda, MD, USA; grant number K23 HL086714).

\section{STATEMENT OF INTEREST}

A statement of interest for this study can be found at

www.erj.ersjournals.com/misc/ statements.dtl 


\begin{tabular}{|c|c|c|}
\hline & OSA & Controls \\
\hline Subjects $n$ & 11 & 10 \\
\hline Age yrs & $40 \pm 14$ & $40 \pm 9$ \\
\hline Male $n$ & 6 & 5 \\
\hline BMI $\mathbf{k g} \cdot \mathrm{m}^{-2}$ & $32 \pm 6$ & $30 \pm 7$ \\
\hline AHI events $\cdot h^{-1}$ & $40 \pm 37$ & $1 \pm 2$ \\
\hline ODI4 events $\cdot h^{-1}$ & $11(0-28)$ & $0(0-0)$ \\
\hline $\mathrm{Sp}, \mathrm{O}_{2}$ nadir $\%$ & $85 \pm 9$ & $96 \pm 3$ \\
\hline Time $\mathrm{Sp}_{\mathrm{p}, \mathrm{O}_{2}}<\mathbf{9 0 \%} \%$ of total sleep time & $1(0-16)$ & $0(0-0)$ \\
\hline ESS & $5 \pm 5$ & $5 \pm 4$ \\
\hline Brachial artery flow-mediated dilation \% & $4 \pm 3$ & $10 \pm 3$ \\
\hline \multicolumn{3}{|c|}{$\begin{array}{l}\text { Data are presented as mean } \pm \mathrm{SD} \text { or median (interquartile range), unless } \\
\text { otherwise stated. OSA: obstructive sleep apnoea; } \mathrm{BMI} \text { : body mass index; } \mathrm{AHI} \text { : } \\
\text { apnoea/hypopnoea index; ODI4: number of arterial oxyhaemoglobin desatura- } \\
\text { tion events } \geqslant 4 \% \text { per hour of sleep; } \mathrm{Sp}, \mathrm{O}_{2} \text { : arterial oxygen saturation measured } \\
\text { by pulse oximetry; ESS: Epworth Sleepiness Scale. }\end{array}$} \\
\hline
\end{tabular}

\section{Study protocol}

All study participants underwent attended nocturnal polysomnography at Columbia University, as previously described [2]. Apnoeas, hypopnoeas and sleep staging were defined as outlined by the American Academy of Sleep Medicine [10]. The Epworth Sleepiness Scale score was calculated as previously described [11]. Blood sample collection and flowmediated dilation (FMD) were performed between 09:0011:00 h, within $48 \mathrm{~h}$ of polysomnography while fasting (see online supplementary material) [12]. Platelet-poor plasma was isolated from citrated blood by centrifugation at $700 \mathrm{rpm}$ for $10 \mathrm{~min}$ followed by centrifugation at 1,800 rpm for $6 \mathrm{~min}$.

\section{KL-6 measurement}

Plasma KL-6 was measured by a sandwich-type ELISA using a KL-6 antibody kit (Sanko Junyaku and Eisai Co. Ltd, Tokyo, Japan; see online supplementary material). The mean \pm SD KL- 6 level in healthy individuals is $239 \pm 102 \mathrm{U} \cdot \mathrm{mL}^{-1}$ with a reference range of $40-438 \mathrm{U} \cdot \mathrm{mL}^{-1}[13]$.

\section{Statistical analysis}

Continuous data are presented as mean \pm SD or median (interquartile range). Categorical data are presented as frequencies. The Wilcoxon rank sum tests and Spearman correlation coefficients were used as appropriate. Potential confounders and mediators of the association between KL-6 levels and OSA were explored by regressing KL-6 level on OSA adjusting for a single covariate using linear regression. In light of the small sample size, partial Spearman correlation coefficients were also used to examine adjusted correlations with KL-6. Since both analyses resulted in similar findings, only the results of the linear models are shown.

Assuming a mean KL-6 level of $239 \mathrm{U} \cdot \mathrm{mL}^{-1}$ among controls and an SD of $102 \mathrm{U} \cdot \mathrm{mL}^{-1}$, it was estimated that there would be $80 \%$ power to detect a mean level of $378 \mathrm{U} \cdot \mathrm{mL}^{-1}$ among those with OSA with $\alpha=0.05$ using a two-sided Wilcoxon rank sum

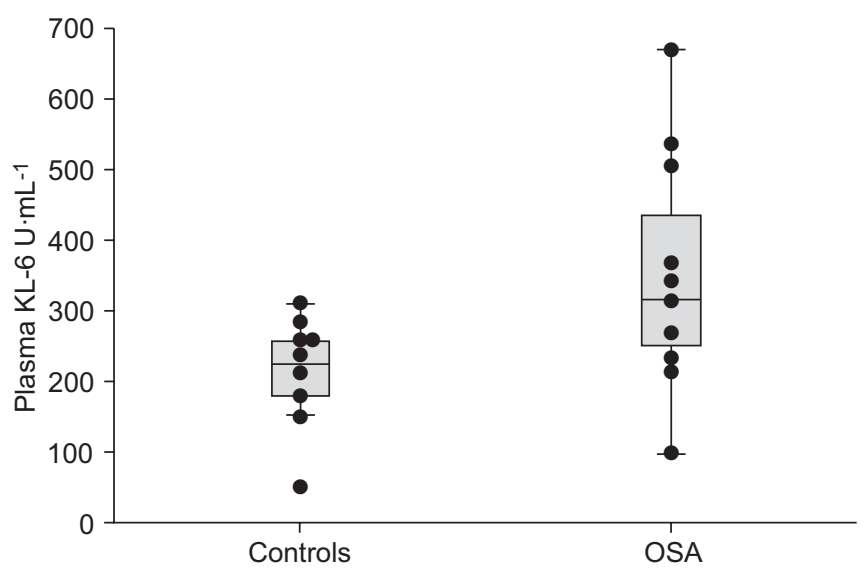

FIGURE 1. Box plots of plasma KL-6 levels in 10 controls and 11 subjects with obstructive sleep apnoea (OSA). The median values and interquartile ranges are represented by the horizontal black lines and the upper and lower bounds of the box, respectively. The whisker tips extend to the most extreme value within 1.5 times the interquartile range. $\bullet$ : individual $\mathrm{KL}-6$ levels for the 21 study subjects. $\mathrm{p}=0.03$ (Wilcoxon rank sum test) for the comparison between groups.

test [13]. Statistical significance was defined as two-tailed p-values $<0.05$.

\section{RESULTS}

In total, 11 out of the 30 subjects with OSA and 10 out of the 15 controls in the prospective study had plasma available for analysis and were included in the current study (see online supplementary material). The mean age of subjects was $40 \pm 12 \mathrm{yrs}$ and $52 \%$ were males. The mean BMI was $31 \pm 6 \mathrm{~kg} \cdot \mathrm{m}^{-2}$. Subjects were well matched for age, sex and BMI (table 1).

The median (interquartile range) plasma KL-6 levels were significantly higher in subjects with OSA compared with

\section{TABLE 2 Spearman correlation coefficients}

\begin{tabular}{lcc} 
Variable & $\begin{array}{c}\text { Spearman correlation } \\
\text { coefficient with plasma } \\
\text { KL-6 level }\end{array}$ & p-value \\
& -0.10 & 0.68 \\
\hline Age & 0.04 & 0.87 \\
BMI & & \\
Polysomnography & 0.61 & 0.003 \\
AHI & 0.49 & 0.02 \\
ODI4 & -0.57 & 0.007 \\
Sp, $\mathrm{O}_{2}$ nadir & 0.53 & 0.01 \\
Time Sp, $\mathrm{O}_{2}<90 \%$ & -0.04 & 0.86 \\
ESS & -0.49 & 0.03 \\
Brachial artery flow- & & \\
mediated dilation & &
\end{tabular}

BMI: body mass index; AHI: apnoea/hypopnoea index; ODI4: number of arterial oxyhaemoglobin desaturation events $\geqslant 4 \%$ per hour of sleep; $\mathrm{Sp}, \mathrm{O}_{2}$ : arterial oxygen saturation measured by pulse oximetry; ESS: Epworth Sleepiness Scale. 

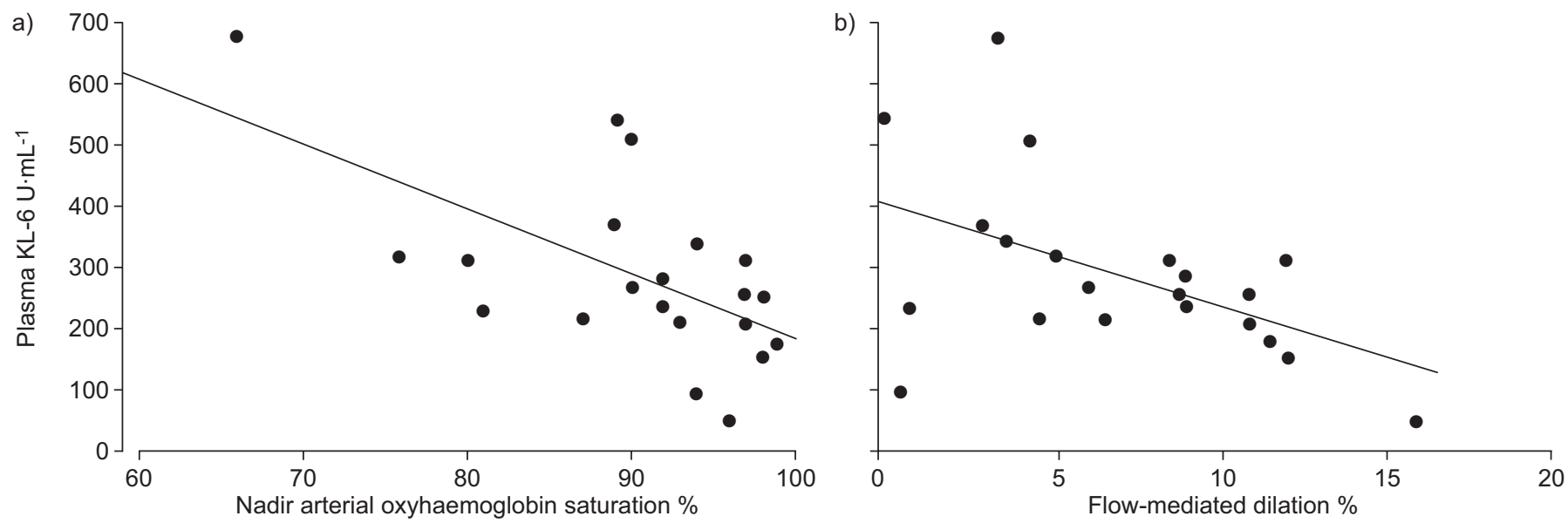

FIGURE 2. Scatter plots of plasma KL-6 level with a) nadir arterial oxyhaemoglobin saturation during sleep and b) brachial artery flow-mediated dilation. Each data point represents one study subject. __ regression lines. a) Spearman correlation coefficient $-0.57 ; p=0.007$. b) Spearman correlation coefficient $-0.49 ; p=0.03$.

controls, $317(232-506) \mathrm{U} \cdot \mathrm{mL}^{-1}$ versus $226(179-257) \mathrm{U} \cdot \mathrm{mL}^{-1}$, respectively ( $\mathrm{p}=0.03$; fig. 1$)$.

A higher plasma KL-6 level was associated with greater AHI, greater time spent with arterial oxygen saturation measured by pulse oximetry $\left(\mathrm{Sp}_{\mathrm{p}} \mathrm{O}_{2}\right)<90 \%$, lower nadir $\mathrm{Sp}_{\mathrm{p}} \mathrm{O}_{2}$, more frequent oxyhaemoglobin desaturation $>4 \%$ per hour of sleep and lower brachial artery FMD (table 2; fig. 2).

The mean increase in KL-6 among subjects with OSA compared with controls was $137 \mathrm{U} \cdot \mathrm{mL}^{-1}(95 \%$ confidence interval $25-245 ; \mathrm{p}=0.03$ ). Adjustment for nadir $\mathrm{S}_{\mathrm{p}, \mathrm{O}_{2}}$ or for FMD attenuated this difference, suggesting that hypoxaemia and/or endothelial dysfunction might have contributed to higher KL-6 levels in OSA (table 3). Nonparametric methods showed similar findings (see online supplementary material).

One control subject had a plasma KL-6 level below the lower limit of detection. When this subject was excluded the results were virtually identical. Similarly, when the subject with the highest KL-6 level $\left(673 \mathrm{U} \cdot \mathrm{mL}^{-1}\right)$ was excluded the results remained significant.

\section{DISCUSSION}

In the current preliminary study, the authors found that some patients with OSA had elevated circulating levels of KL-6, a marker of increased alveolar wall permeability, compared with age-, sex- and BMI-matched controls. KL-6 levels correlated strongly with nocturnal hypoxaemia and peripheral endothelial dysfunction, as measured by brachial artery FMD. These same factors appeared to explain, at least in part, the higher levels of KL-6 in OSA patients.

The present results suggest that alveolar wall permeability may be increased in OSA. One mechanism by which OSA could increase the permeability of the alveolar wall is through the generation of oxidative stress arising from cyclic local hypoxia and reoxygenation in the alveolus. Such intermittent hypoxia has been implicated as a contributor to vascular and systemic inflammation in OSA $[2,14]$. In the present study, greater nocturnal hypoxaemia was associated with higher circulating KL-6 levels, and the degree of nocturnal hypoxaemia appeared to explain at least some of the elevation in
KL-6 in OSA. The current results must be interpreted cautiously given the small sample size. Nevertheless, the results suggest a role for local intermittent hypoxia in the development of increased alveolar wall permeability in OSA. Future studies should examine the relative contributions of intermittent and continuous hypoxia on circulating KL-6 levels in OSA and chronic lung diseases, such as chronic obstructive pulmonary disease.

In addition to hypoxia-reoxygenation, mechanical stretch is an intuitively appealing mechanism of epithelial or endothelial cell injury in the lung. Dynamic changes in intrathoracic pressure (Mueller manoeuvres) accompany obstructive apnoeas and hypopnoeas and could conceivably deform alveolar epithelial cells and endothelial cells, mirroring the changes seen in ventilator-induced lung injury [15] and

\begin{tabular}{|c|c|c|c|c|}
\hline \multirow{2}{*}{$\begin{array}{l}\text { TABLE } 3 \\
\text { Model }\end{array}$} & \multicolumn{4}{|c|}{$\begin{array}{l}\text { Unadjusted and adjusted mean increases in } \\
\text { plasma KL-6 level in subjects with obstructive } \\
\text { sleep apnoea compared with controls }\end{array}$} \\
\hline & & $\begin{array}{l}\text { Mean increase in } \\
\text { plasma } \mathrm{KL}-6 \mathrm{U} \cdot \mathrm{mL}^{-1}\end{array}$ & $95 \% \mathrm{Cl}$ & $p$-value \\
\hline \multicolumn{2}{|l|}{ Unadjusted } & 137 & $25-245$ & 0.03 \\
\hline \multicolumn{5}{|l|}{ Adjusted } \\
\hline \multicolumn{2}{|l|}{ Age } & 136 & $24-248$ & 0.03 \\
\hline \multicolumn{2}{|l|}{ Sex } & 139 & $27-251$ & 0.03 \\
\hline \multicolumn{2}{|l|}{ BMI } & 133 & $17-149$ & 0.04 \\
\hline \multicolumn{2}{|l|}{ ODI4 } & 153 & $25-280$ & 0.03 \\
\hline \multicolumn{2}{|l|}{$\mathrm{Sp}, \mathrm{O}_{2}$ nadir } & 42 & $-95-179$ & 0.56 \\
\hline \multicolumn{2}{|c|}{ Time $\mathrm{Sp}, \mathrm{O}_{2}<90 \%$} & 115 & $-8-239$ & 0.08 \\
\hline \multicolumn{2}{|c|}{ ESS } & 137 & 23-251 & 0.03 \\
\hline \multicolumn{2}{|c|}{$\begin{array}{l}\text { Brachial artery flow- } \\
\text { mediated dilation }\end{array}$} & 75 & $-92-242$ & 0.39 \\
\hline
\end{tabular}


perhaps leading to stress failure of endothelial cells [16]. Measurement of circulating KL-6 levels in healthy controls before and after Mueller manoeuvres under normoxic and hypoxic conditions may shed light on the injurious role of mechanical stretch in OSA in future studies.

The significance of the association between higher circulating KL-6 levels and greater endothelial dysfunction in the brachial artery is unclear. One explanation might be a common antecedent cause of both alveolar injury and peripheral endothelial injury, such as oxidative stress. Alternatively, this association may signal a role for pulmonary endothelial dysfunction and/or inflammation in the development of increased alveolar wall permeability in OSA. Additional studies of pulmonary endothelial dysfunction and injury in OSA may clarify this finding.

The present results suggest that OSA may be a novel risk factor for subclinical lung injury. The consequences of such injury repeated nightly over many years are unknown. For susceptible individuals, it can be speculated that such injury might be followed by abnormal wound healing and local fibrosis in areas prone to local hypoxia and mechanical stretch during a Mueller manoeuvre. Intriguingly, an association between OSA and idiopathic pulmonary fibrosis, a disease characterised by peripheral lower lobe fibrosis, has been reported recently [17]. The current findings should prompt further study of the mechanisms (and confounders) of this association.

The present study had several limitations. First and foremost, only 11 patients with OSA were examined at a single centre, making chance and selection bias plausible explanations for the results. However, the cohort was recruited from consecutive patients with suspected sleep disordered breathing, and circulating KL-6 levels were associated with nocturnal hypoxaemia and endothelial dysfunction in mechanistically plausible directions concordant with the a priori hypothesis. Nevertheless, the current results should be confirmed in a larger cohort with greater power to perform multivariate adjustment and examine markers of epithelial and endothelial injury and function. Secondly, alveolar wall permeability was not directly measured. Instead, the present authors relied on a circulating biomarker that has consistently been shown to be elevated in those with lung injury. Finally, the increase in circulating KL-6 in OSA patients was modest, albeit in a range similar to that of acute lung injury survivors [7].

In summary, elevated circulating levels of KL-6 were detected in patients with obstructive sleep apnoea, suggesting that alveolar wall permeability might be increased in obstructive sleep apnoea. Additional studies should focus on determining the site, mechanisms and consequences of this finding.

\section{ACKNOWLEDGEMENTS}

The authors would like to acknowledge Sanko Junyaku and Eisai Co. Ltd (Tokyo, Japan) for kindly performing the KL-6 assays.

\section{REFERENCES}

1 Basner RC. Continuous positive airway pressure for obstructive sleep apnea. N Engl J Med 2007; 356: 1751-1758.
2 Jelic S, Padeletti M, Kawut SM, et al. Inflammation, oxidative stress, and repair capacity of the vascular endothelium in obstructive sleep apnea. Circulation 2008; 117: 2270-2278.

3 Kiefmann R, Rifkind JM, Nagababu E, Bhattacharya J. Red blood cells induce hypoxic lung inflammation. Blood 2008; 111: 4831-4832.

4 Cochrane CG, Spragg R, Revak SD. Pathogenesis of the adult respiratory distress syndrome. Evidence of oxidant activity in bronchoalveolar lavage fluid. J Clin Invest 1983; 71: 754-761.

5 Fletcher EC, Proctor M, Yu J, et al. Pulmonary edema develops after recurrent obstructive apneas. Am J Respir Crit Care Med 1999; 160: 1688-1696.

6 Chaudhary BA, Ferguson DS, Speir WA Jr. Pulmonary edema as a presenting feature of sleep apnea syndrome. Chest 1982; 82: 122-124.

7 Ishizaka A, Matsuda T, Albertine KH, et al. Elevation of KL-6, a lung epithelial cell marker, in plasma and epithelial lining fluid in acute respiratory distress syndrome. Am J Physiol Lung Cell Mol Physiol 2004; 286: L1088-L1094.

8 Nathani N, Perkins GD, Tunnicliffe W, Murphy N, Manji M, Thickett DR. Kerbs von Lungren 6 antigen is a marker of alveolar inflammation but not of infection in patients with acute respiratory distress syndrome. Crit Care 2008; 12: R12.

9 Sato H, Callister ME, Mumby S, et al. KL-6 levels are elevated in plasma from patients with acute respiratory distress syndrome. Eur Respir J 2004; 23: 142-145.

10 Iber C, Ancoli-Israel S, Chesson A, Quan SF. The AASM Manual for the Scoring of Sleep and Associated Events: Rules, Terminology, and Technical Specifications. 1st Edn. Westchester, American Academy of Sleep Medicine, 2007.

11 Johns MW. A new method for measuring daytime sleepiness: the Epworth Sleepiness Scale. Sleep 1991; 14: 540-545.

12 Corretti MC, Anderson TJ, Benjamin EJ, et al. Guidelines for the ultrasound assessment of endothelial-dependent flow-mediated vasodilation of the brachial artery: a report of the International Brachial Artery Reactivity Task Force. J Am Coll Cardiol 2002; 39: 257-265.

13 Janssen R, Kruit A, Grutters JC, Ruven HJ, Gerritsen WB, van den Bosch JM. The mucin-1 568 adenosine to guanine polymorphism influences serum Krebs von den Lungen-6 levels. Am J Respir Cell Mol Biol 2006; 34: 496-499.

14 Savransky V, Nanayakkara A, Li J, et al. Chronic intermittent hypoxia induces atherosclerosis. Am J Respir Crit Care Med 2007; 175: 1290-1297.

15 Tschumperlin DJ, Oswari J, Margulies AS. Deformationinduced injury of alveolar epithelial cells. Effect of frequency, duration, and amplitude. Am J Respir Crit Care Med 2000; 162: 357-362.

16 West JB, Mathieu-Costello O. Stress failure of pulmonary capillaries: role in lung and heart disease. Lancet 1992; 340: 762-767.

17 Lancaster LH, Mason WR, Parnell JA, et al. Obstructive sleep apnea is common in IPF. Am J Respir Crit Care Med 2008; 177: Suppl., A247. 